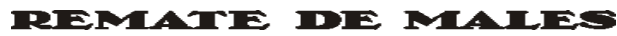

Campinas-SP, v. 38, n. 1, pp. 1-9, jan./jun. 2018

\title{
E ENTÃo, POR QUE O ÍNDICO?
}

Por detrás de tantos nomes e tantos cruzamentos, de tanta diversidade, é sempre o mesmo, o mar.

(João Paulo Borges Coelho)

O dossiê temático "Espaços transnacionais: narrativas do Oceano Índico" que aqui se apresenta constitui um dos resultados das pesquisas e das colaborações científicas e institucionais desenvolvidas no âmbito de dois projetos atualmente em curso na Universidade de Lisboa (Portugal) e na Universidade Estadual de Campinas (Brasil). ${ }^{1}$ Em ambos os casos os projetos se situam na área dos Estudos do Oceano Índico (Indian Ocean Studies), desdobrando-se em diversos campos do saber, e incluem o estudo e a análise cultural de diversos contextos geográficos e espaciais, nomeadamente: África, Ásia e Europa.

No que diz respeito ao Projeto NILUS (Projeto FCT, Ref. PTDC/ CPCELT/4868/2014), a pesquisa investiga de que modo as narrativas escritas e visuais - oriundas de contextos lusófonos, contribuem para a construção de um imaginário transnacional do Oceano Índico, onde os universos de língua portuguesa aparecem todavia como marginais e/ou minoritários. Por conseguinte, através de um mapeamento de

1 Projeto de Investigação “NILUS - Narrativas do Oceano Índico no Espaço Lusófono” (Ref. PTDC/CPCELT/4868/2014), financiado pela Fundação para a Ciência e a Tecnologia - FCT (Portugal) sob coordenação de Ana Mafalda Leite e desenvolvido no Centro de Estudos sobre Âfrica, Ásia e América Latina CEsA - ISEG da Universidade de Lisboa - UL; e Projeto de Pesquisa Fapesp “A Estética do Índico. 'Geografias Transnacionais do Imaginário' em narrativas visuais e literárias na(s) África(s) contemporânea(s)” (REF: 2016/26098-5), desenvolvido por Elena Brugioni (Pesquisadora Responsável) e Jéssica Bezerra da Silva (Bolsista de Treinamento Técnico Fapesp, REF. 2017/12764-6) no Departamento de Teoria Literária da Universidade Estadual de Campinas - Unicamp. 
narrativas literárias e representações visuais que foram produzidas em Portugal, Moçambique, Goa e Timor Leste a partir do século XX até à contemporaneidade, o Projeto procura mapear uma arqueologia desse imaginário para refletir crítica e comparativamente sobre as suas atuais configurações, analisando o papel da narrativa nos processos de construção identitária no espaço regional e transnacional do Oceano Índico. Tanto na recolha, quanto na análise das narrativas literárias e visuais, o Projeto desenvolve-se em torno de diversos eixos temáticos, tais como: as representações do espaço (ilhas, cidades portuárias e costeiras, lugares do comércio etc.); as representações dos povos (Indianos, Africanos, Timorenses etc.) e das suas experiências, individuais e colectivas, de migração e deslocamento; as representações de objectos, comida, vestuário, encarando a cultura material como elemento distintivo do imaginário transnacional do Oceano Índico.

Por outro lado, o projeto sobre "A Estética do Indico" (Projeto FAPESP, Ref. 2016/26098-5) pretende analisar diversas práticas de representação literatura, fotografia documental e artes visuais - em diferentes contextos da África subsaariana - Moçambique, Djibouti, Somália, Quénia, Tanzânia, Madagascar, Maurícias, entre outros -, encarando o Oceano Índico como um paradigma crítico transnacional (HOFMEYR, 2007) e, simultaneamente, como uma "geografia transnacional do imaginário" (GHOSH; MUECKE, 2007), encarando as representações do Índico Africano (ALPERS, 2009) como "práticas de produção e capitalização do conhecimento" (HOUNTONDJI, 1997; 2002) e, logo, na perspectiva de "experiências estéticas e epistemológicas" (CANCLINI, 2012) matriciais para (re)pensar a gramática crítica e os discursos identitários e culturais da contemporaneidade pós-colonial.

Realçando a diversidade do corpus analisado e dos contextos em torno dos quais as pesquisas se desenvolvem, os dois projetos partem de abordagens e inquietações crítico-teóricas comuns suscitadas, em primeiro lugar, pela ausência da perspectiva crítica e conceitual que pauta os Estudos do Oceano Índico na análise literária, visual e cultural em contextos de língua portuguesa - África, Brasil, Europa - onde esse espaço transnacional permanece todavia ausente ou escassamente consolidado, quando não refém de narrativas imperiais tão celebratórias quanto parciais e problemáticas. Surge, em segundo lugar, a necessidade de reformular paradigmas críticos, capazes de sustentar e reposicionar a prática humanista como conhecimento e manancial teórico indispensável 
para decifrar os indícios e os desafios de um mundo cada vez mais abalado pelas suas "múltiplas desordens pós-coloniais" (SASSEN, 2000).

Nesse sentido, os Estudos do Oceano Índico - a entender-se como espaço físico, estético e político, desdobrado em diversas latitudes e representações - oferecem novas possibilidades epistemológicas e interpretativas, críticas e conceituais, contribuindo substancialmente para evidenciar movimentos, deslocações, universalismos e cosmopolitismos, frequentemente marginalizados por narrativas e abordagens de matriz colonial, nacional ou pós-imperial. Para além de um aparato crítico inédito e pertinente de um ponto de vista operacional, o Oceano Índico proporciona também o surgimento de perspetivas transnacionais situadas, ou melhor, daquilo que pode ser definido como "transnacionalismos menores" (LIONNET; SHIH, 2005), em que a ênfase crítica recai sobre os movimentos transversais e minoritários da(s) cultura(s) - tal como é o caso da "revisão transcolonial" de Shakespeare em contextos africanos póscoloniais, analisada por Lionnet (2005, pp. 206-207) - e, desse modo, aponta para perspetivas distintas das narrativas da flexibilidade e do nomadismo que caracterizam a vulgata pós-colonial (LIONNET; SHIH, 2005, pp. 8-9). Assim sendo, os Estudos do Índico desdobrados na perspetivas do(s) transnacionalismo(s) menor(es) contribuem sensivelmente para redefinir a mesma noção de pós-colonialidade, apontando para discursos críticos capazes de desmantelar o cariz hegemónico do pós-colonial como abordagem assombrada pelo feiticismo da alteridade e pelo seu desengajamento das condições materiais do literário, do artístico e do cultural (AHMAD, 2002; LAZARUS, 2011).

Por conseguinte surge não apenas uma perspetivação crítica inovadora, de um ponto de vista conceitual, bem como analítico, e sem ambições totalizantes, mas também um campo de estudo que se pauta pela possibilidade de reconfigurar momentos, sujeitos e práticas de representação “em contraponto" (SAID, 1993), instituindo-se como um gesto crítico irremediavelmente ligado às formas de ver e imaginar o mundo (SAID, 1993) e apontando a um passo crucial para renovar as potencialidades interpretativas e conceptuais dos estudos comparados numa perspetiva pós-colonial (SPIVAK, 2003), bem como da própria prática humanística (SAID, 2004).

Impossível ilustrar os debates e as pistas teóricas que os Estudos do Índico proporcionam e de que esse Dossiê, embora de modo seletivo, pretende dar conta. Cabe no entanto realçar, apesar dos limites a que este 
breve ensaio introdutório precisa obedecer, algum dos eixos conceituais que norteiam nossa reflexão, rastreando itinerários para novos e futuros desafios que as águas mansas e misteriosas do Índico obrigarão a enfrentar. Olhando pelo conjunto dos ensaios que aqui se reúnem, diversos e complementares ao mesmo tempo, sobressai de forma evidente e inequívoca a dimensão diferencial que o Índico institui e significa, desdobrando-se em vários âmbitos e, em última instância, configurando perspetivas espaciais, imaginários e conceitos que não podem ser descritos por nenhuma teoria e abordagem de cariz totalizante. Diferencial como diferença, apontando para a célebre reflexão desenvolvida por Jaques Derrida (1967) e salientando, desse modo, a dimensão de uma construção conceitual, metodológica e disciplinar incapaz de ser completamente descrita por qualquer estrutura.

Daí a ambiguidade conceitual da mesma noção subjacente à geografia do Oceano Índico: região, sistema-mundo, arena, espaço transnacional, desenhando uma constelação crítica e conceitual instável e múltipla, mas sobretudo reveladora da natureza produtivamente ambígua e plástica do Índico como paradigma analítico e como geografia transnacional, refundadora para as práticas de narrar e imaginar que os ensaios aqui reunidos em geral abordam. Para além disso, situa-se a dimensão utópica que essas narrativas e esses imaginários sobre o Oceano Índico em geral contêm e significam. Uma utopia que parece configurar o Índico como uma "geografia reconquistada" (SAID, 1993), um espaço estético e político libertado do discurso imperial, cuja dimensão transnacional permite também repensar os limites - individuais e colectivos - do espaço da nação, apontando para aquilo que Philip E. Steinberg (2013) define como "aquatopia”, salientando a dimensão emancipatória e libertadora da utopia que se articula através de narrativas e imaginários marítimos e oceânicos, e logo configurando o espaço líquido do mar e do oceano como "uma construção social, literária e cultural" (STEINBERG, 2001).

Na aproximação aqui proposta entre o Oceano Índico e o chamado "espaço lusófono" - outra realidade per se inatingível, outra geografia complexa, traçada pela herança do colonialismo -, destaca-se, geográfica e criticamente, Moçambique, com suas narrativas e suas margens mais declaradamente índicas, como as ilhas, as cidades costeiras e portuárias. De facto, foi a partir da configuração crítica de uma vertente índica da literatura moçambicana que arrancaram os projetos e as reflexões em torno de um Oceano Índico cruzado pela língua portuguesa, que aqui 
se apresentam já em diálogo com outros espaços e outras línguas. Mas é preciso dizer que, principalmente no domínio dos estudos literários e culturais - já que a historiografia se vem soltando mais agilmente de amarras político-ideológicas -, situar Moçambique no Oceano Índico é ainda um gesto controverso, suscetível de traçar terrenos movediços e sensíveis; pelas suspeitas de um suposto "branqueamento" do imaginário africano, acarretado pela perspectiva índica; pelo risco fácil de se equacionar esse tipo de abordagem com uma celebração acrítica de uma identidade colectiva, quando um dos seus traços marcantes, pelo contrário, se confronta criticamente na relação com as "origens" e com o "Outro".

No entanto, situar Moçambique nessa cartografia outra significa aceitar um desafio incontornável para os Estudos do Oceano Índico: refletir de forma mais abrangente sobre as relações entre a costa africana e o espaço inter-regional do Índico, necessidade apontada tanto no domínio da historiografia (BOSE, 2006; PEARSON, 2003 e 2011; entre outros) quanto na área dos estudos literários e culturais (HOFMEYR, 2007; SAMUELSON, 2010; ADEJUNMOBI, 2009). Por outro lado, tendo em conta as especificidades da ocupação portuguesa no território moçambicano, a articulação proposta pelos projetos mencionados e pelas reflexões aqui reunidas, pretende propor uma releitura do Moçambique independente à luz da emergência de "cosmpolitismos locais", produzidos pelas movimentações no espaço do Oceano Índico (MENESES, 2012).

Aceitando e assumindo o percurso e o desafio lançado pelo escritor moçambicano João Paulo Borges Coelho em suas recolhas de contos intituladas Índicos indícios (2005), propomos também recolhas e análises de indícios e fragmentos de narrativas do Oceano Índico ainda por desvendar e articular. Trata-se de narrativas suscetíveis de constituírem um corpus transnacional capaz de reconstruir as múltiplas e possíveis imagens de um Oceano Índico em constante mudança e movimento.

Entre os movimentos teóricos e críticos aqui propostos, esboçase uma operação epistemológica que pretende articular tanto uma cartografia "lusófona" do Oceano Índico - necessariamente em diálogo com os outros espaços culturais presentes - quanto uma cartografia índica do chamado espaço lusófono, vocacionada para iluminar histórias, narrativas e relações ainda pouco exploradas, já que em diversos domínios (como também nos estudos literários), esse espaço acaba por ser uma projeção de unidades isoladas, e não uma rede de conhecimentos e trocas. 
Esse tipo de operação cartográfica pretende tanto ultrapassar o isolamento "linguístico" que por vezes afecta os países de língua portuguesa, quanto vincar a contribuição do imaginário declinado em língua portuguesa para um mais amplo imaginário transnacional e translinguístico do Índico. Pretende assim reequacionar tanto a passada narrativa hegemónica do colonialismo português quanto a presente narrativa da Lusofonia, a partir da reconstituição de narrativas que dão conta de conflitos e resistências, de heranças culturais múltiplas, de memórias partilhadas e divididas.

“E então por que o Índico?" - o título da nossa apresentação retoma assim algumas das questões que os vários ensaios desse dossiê ensaiam problematizar, e gostaríamos de realçar a importância da tradução do ensaio introdutório de Isabel Hofmeyr, aqui publicado "O Atlântico negro encontra o Oceano Índico: forjando novos paradigmas do transnacionalismo para o Sul global - perspectivas literárias e culturais", que é ponto de partida de um outro trabalho dos nossos projectos de pesquisa, nomeadamente a criação de uma antologia de textos teóricos sobre os Estudos do Oceano Índico, traduzidos para o português.

A estudiosa sul-africana no seu texto articula sabiamente a dupla dimensão narrativa sobre o Índico, a histórica e a literária, mostrando sua interacção e fundamentando seu estudo numa bibliografia de referência fundamental. Refere alguns dos temas unificadores e recorrentes do IOW (Indian Ocean World - Mundo do Oceano Índico), como o comércio, o capital, o trabalho, a religião, as cidades portuárias, as viagens e diásporas, a guerra, o domínio colonial, os movimentos anticoloniais. Ilhas, pessoas e passagens são outros tópicos desenvolvidos nesse ensaio nuclear do dossiê, que o leitor de imediato irá ler. Segundo Isabel Hofmeyr, as ilhas seriam fundamentais para a formulação de novos circuitos literários, que as diásporas e viagens narrativizam em romance e História. Há laços comparativos de estéticas e tradições a estudar sobre as literaturas das várias ilhas do Oceano Índico e suas relações com os diversos espaços continentais, como África, Índia, Indonésia e vice-versa, em cartografias que exigem dos investigadores a seriedade criativa de viajantes entre teoria e análise narrativa.

Alertando ainda para o perigo das generalizações, o ensaio da autora sul-africana mostra-nos que a importância dos estudos sobre o mundo do Oceano Índico é também a da análise dos seus arquivos: "precisamos pensar o Oceano Índico como o local por excelência das 'modernidades 
alternativas'; formações da modernidade que tomaram forma em um arquivo de tradições sociais e intelectuais profundas e articuladas".

Cabem por fim, mas não por obedecer a uma ordem de importância, umas palavras de agradecimento a todas as pessoas envolvidas na publicação desse Dossiê, que marca, para além de um passo fundamental para as pesquisas e os projetos a ele ligados, também uma partilha e um diálogo académico, intelectual e humano tão importante quanto, cada vez mais, raro e precioso. Desse modo, os nossos mais sinceros agradecimentos são dirigidos às equipas dos projetos NILUS e A Estética do Índico, cujo trabalho, empenho e participação foram vitais para que com esta publicação se alcançasse hoje um importante resultado das pesquisas até agora desenvolvidas; aos autores e às autoras dos ensaios aqui reunidos que com seus trabalhos e reflexões tornaram essa travessia do Oceano Índico ainda mais rica e articulada; e aos editores da revista Remate de Males, que acolheram nossa proposta e com grande dedicação e competência tornaram possível a publicação do Dossiê "Espaços transnacionais: narrativas do Oceano Índico".

Ana Mafalda Leite ${ }^{2}$ Jessica Falconi ${ }^{3}$ Elena Brugioni ${ }^{4}$

\section{REFERÊNCIAS}

ADEJUNMOBI, Moradewun. Claiming the Field. Africa and the Space of Indian Ocean Literature. Callaloo, v. 32, n. 4, 2009, pp. 1.247-1.261.

AHMAD, Aijaz. Linhagens do presente. Ensaios. São Paulo: Boitempo Editorial, 2002.

ALPERS, Edward. East Africa and the Indian Ocean. Princeton: Markus Wiener Publishers, 2009.

2 Ana Mafalda Leite é Professora Associada com Agregação da Faculdade de Letras da Universidade de Lisboa na área de Estudos Africanos e investigadora do Centro de Estudos sobre África, Ásia e América Latina CEsA - ISEG, Portugal: anamafaldaleite@gmail.com. 3 Jessica Falconi é pesquisadora do Centro de Estudos sobre África, Ásia e América Latina CEsA - ISEG da Universidade de Lisboa, Portugal, e Professora Visitante na Universidade Autónoma de Barcelona e responsável pelo Centro de Língua Portuguesa/IP Camões na mesma instituição, Espanha: jessica-77@libero.it.

4 Elena Brugioni é professora de Literaturas Africanas e Estudos Pós-coloniais no Departamento de Teoria Literária do Instituto de Estudos da Linguagem da Universidade Estadual de Campinas, Unicamp: elena@iel.unicamp.br. 
BORGES COELHO, João Paulo. Índicos Indícios I - Setentrião. Lisboa: Caminho, 2005.

BOSE, Sugata. A Hundred Horizons: The Indian Ocean in an Age of Global Empire. Cambridge, Mass.: Harvard University Press, 2006.

DERRIDA, Jaques. De la grammatologie. Paris: Éditions de Minuit, 1967.

GARCÍA CANCLINI, Néstor. A sociedade sem relato. Antropologia e Estética da Iminência. São Paulo: Edusp, 2012.

GHOSH, Devleena; MUECKE, Stephen (Eds.). Cultures of Trade: Indian Ocean Exchanges. Newcastle: Cambridge Scholars Publishing, 2007.

HOFMEYR, Isabel. The Black Atlantic Meets the Indian Ocean: Forging New Paradigms for Transnationalism for the Global South. Literary and Cultural Perspectives. Social Dynamics: A Journal of African Studies, v. 33, n. 2, 2007, pp. 3-32.

HOUNTONDJI, Paulin. (Ed.). Endogenous Knowledge: Research Trails. Dakar: Codesria, 1997.

HOUNTONDJI, Paulin. The Struggle for Meaning: Reflections on Philosophy, Culture, and Democracy in Africa. Athens: Ohio University Center for International Studies, 2002.

LAZARUS, Neil. What Postcolonial Theory doesn't Say. Race E Class, v. 53, n. 1, 2011, pp. 3-27.

LIONNET, Françoise. Transcolonial Translations: Shakespeare in Mauritius. In: LIONNET, Françoise; SHIH, Shu-mei. Minor Transnationalism. Durham e Londres: Duke University Press, 2005, pp. 201-222.

LIONNET, Françoise; SHIH, Shu-mei. Minor Transnationalism. Durham/Londres: Duke University Press, 2005 .

MENESES, Maria Paula. Nação e narrativas pós-coloniais: interrogações em torno dos processos identitários em Moçambique. In: LEITE, Ana Mafalda et al. (Eds.). Nação e narrativa Pós-Colonial I. Angola e Moçambique. Ensaios. Lisboa: Colibri, 2012, pp. 311-322.

PEARSON, Michael N. The Indian Ocean. Londres: Routledge, 2003.

PEARSON, Michael N. History of the Indian Ocean: A Review Essay. Wasafiri, v. 26, n. 2, 2011, pp. 78-99.

SAMUELSON, Meg. Making Home on the Indian Ocean Rim. Relocations in South African Literatures. In: MOORTHY, Shanti; JAMAL, Ashraf (Eds.). Indian Ocean Studies: Cultural, Social, and Political Perspectives. New York: Routledge, 2010, pp. 298-317.

SASSEN, Saskia. Spatialities and Temporalities of the Global: Elements for a Theorization. Public Culture, v. 12, n. 1, 2000, pp. 215-232. 
Remate de Males, Campinas-SP, v. 38, n. 1, pp. 1-9, jan./jun. 2018 - 9

SAID, Edward W. Culture and Imperialism. Nova York: Vintage Books, 1993.

SAID, Edward W. Humanism and Democratic Criticism. Nova York: Palgrave, 2004.

SPIVAK, Gayatri Chakravorty. Death of a Discipline. Nova York: Columbia University Press, 2003.

STEINBERG, Philip E. The Social Construction of the Ocean. Cambridge: Cambridge University Press, 2001.

STEINBERG, Philip E. Comentador Convidado (Guest Commentator). Aquatopia Exhibit, Nottingham Contemporary, 2013. 